\title{
Une photopile à haut rendement pour utilisation spatiale
}

\author{
M. Le Métayer, G. Keil, A. Cuquel et J. Bozec \\ Centre National d'Etudes Spatiales, 18, avenue Edouard Belin, 31055 Toulouse Cedex, France
}

(Reçu le 25 janvier 1982, révisé le 9 avril 1982, accepté le 14 avril 1982)

\begin{abstract}
Résumé. - L'épitaxie en phase vapeur d'arséniure de gallium à partir de composés organométalliques se prête bien à la réalisation de piles solaires. On étudie par cette méthode une homojonction d'arséniure de gallium dont la zone avant est suffisamment fine (de l'ordre de $500 \AA$ ) pour que la majorité des porteurs soit créée sous la jonction; les pertes par recombinaison en surface sont ainsi minimisées.

Les résultats déjà obtenus font ressortir un rendement photovoltaïque proche de $20 \%$ sous un spectre solaire AM1,5 et les premiers essais sous irradiation indiquent un bon comportement en ambiance spatiale.

Abstract. - The method of growing GaAs by pyrolisis of an organic compound of gallium and arsine is well adapted to the fabrication of solar cell devices; we have studied the growth by this method of a GaAs homostructure with a shallow junction. The thickness of the front face is enough to reduce the surface carrier recombination. The best device gives a value of about $20 \%$ conversion efficiency at AM1,5 solar spectrum and the first result under space radiations indicates that such cells are resistant to this type of environment.
\end{abstract}

1. Introdution. L'évolution des programmes spatiaux laišse prévoir un accroissement rapide de la puissance requise pour l'alimentation en énergie des satellites. Pour s'affranchir en partie des problèmes liés à l'augmentation de la surface des générateurs solaires, le développement de photopiles à rendement amélioré est indispensable.

Dans l'état actuel des techniques de fabrication des photopiles, le silicium est le matériau semiconducteur qui offre les meilleures performances. Cependant, ces dernières années, on constate un plafonnement des performances électriques et les structures les plus sophistiquées, obtenues en laboratoire, donnent un rendement de conversion de l'ordre de $15 \%$; en production, le rendement photovoltaïque moyen avoisine les $12 \%$. De plus, pour les applications spatiales, ce matériau présente un grave inconvénient : il se dégrade fortement sous irradiations. Ainsi, le rendement de conversion des piles solaires en fin de vie d'un satellite peut baisser jusqu'à $6 \%$.

Pour ces différentes raisons, les laboratoires travaillant dans les techniques spatiales cherchent à remplacer le silicium.

Un autre semiconducteur, l'arséniure de gallium, donne déjà de très bons résultats. Les Sociétés IBM [1] ou Hughes Research Laboratories [2], avec une technique classique d'élaboration de ce matériau, l'épitaxie en phase liquide, ont déjà réalisé en laboratoire des structures photovoltaiques qui donnent des rendements supérieurs à $18 \%$ sous un éclairement solaire hors atmosphère. En outre, de récentes études comparatives de la NASA [3] et du MIT [4] montrent une meilleure tenue en irradiation des piles solaires réalisées avec l'arséniure de gallium.

Une nouvelle méthode d'épitaxie en phase vapeur de l'arséniure de gallium par pyrolyse d'organométalliques a été proposée en 1968 par Manasevit [5]. Ce procédé, qui a déjà permis aux chercheurs de Rockwell [6] et Varian [7] de réaliser des photopiles à haut rendement, est étudié depuis quelques années dans nos laboratoires $[8,9]$.

Les épitaxies sont réalisées sous pression réduite dans un réacteur en quartz horizontal (Fig. 1). Les substrats sont disposés sur un suscepteur en graphite chauffé par induction haute fréquence; le gaz vecteur, l'hydrogène, amène les composés, le triméthylgallium et l'arsine, dans le réacteur où ils se mélangent et la réaction intervient au voisinage immédiat du substrat. L'épitaxie est réalisée à une vitesse de $0,1 \mu \mathrm{m} / \mathrm{min}$. pour une température de $650^{\circ} \mathrm{C}$; les éléments dopants sont obtenus à partir du diéthylzinc pour le type $P$ et du germane, du tétraméthylétain ou de l'hydrogène sélénié pour le type $\mathbf{N}$.

Nous présentons dans ce papier les résultats obtenus sur des piles solaires réalisées par une telle méthode. 


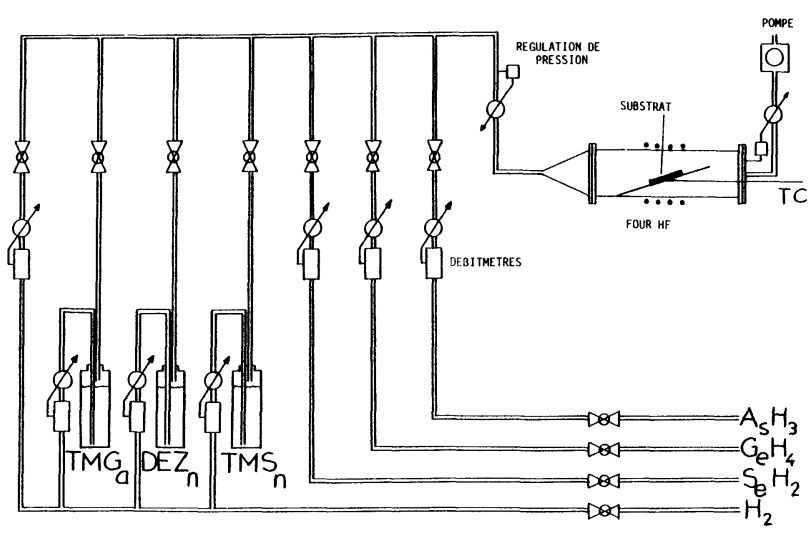

Fig. 1. - Synoptique de l'installation.

[Experimental apparatus.]

2. Description de la structure photovoltaique. La structure de la photopile est choisie en fonction des contraintes imposées par l'ambiance spatiale. En particulier, nous nous sommes rapidement orientés vers des modèles à jonction proche de la surface dont la première réalisation est due à Bozler et Fan en 1977 [10] et qui offrent une plus grande résistance à l'action des électrons et des protons piégés dans la magnétosphère [4]; le choix d'une structure $\mathrm{N} / \mathrm{P}$ ou $\mathrm{P} / \mathrm{N}$ n'est pas encore définitivement arrêté et résultera des essais d'irradiations.

La réalisation d'une telle pile dont la zone avant est très fine pose des problèmes technologiques certains, liés essentiellement au contrôle de la croissance de faibles épaisseurs et aux prises de contacts, mais offre en retour un avantage non négligeable en évitant le recours à une fenêtre d'arséniure de gallium et d'aluminium. En effet, l'inconvénient de l'arséniure de gallium est de présenter un coefficient d'absorption très élevé (qui conduira en particulier les photons d'énergie supérieure à $3 \mathrm{eV}$ à être absorbés pour moitié sur une profondeur d'environ $300 \AA$ ) associé à une vitesse de recombinaison de surface importante, interdisant la collection des charges ainsi créées. Une photopile à l'AsGa, conçue comme les photopiles classiques au silicium (jonction à environ $0,4 \mu \mathrm{m}$ de la surface), ne pourrait donc pas convertir la partie " violette " du spectre solaire.

Face à ce problème, la solution habituellement retenue consiste à diminuer la vitesse de recombinaison de surface en déposant sur la photopile une couche de GaAlAs : la vitesse de recombinaison à l'interface est beaucoup plus faible qu'en surface de l'AsGa.

Une autre méthode consiste à amener les photons à être absorbés après la jonction en approchant celle-ci de la surface : les charges créées lors de l'absorption ne sont donc plus concernées par les phénomènes de surface. Cet effet de la réduction de la profondeur de jonction est illustré par la figure 2 où nous avons porté le rendement de collecte calculé pour différentes photopiles. La technique d'épitaxie aux organométal-

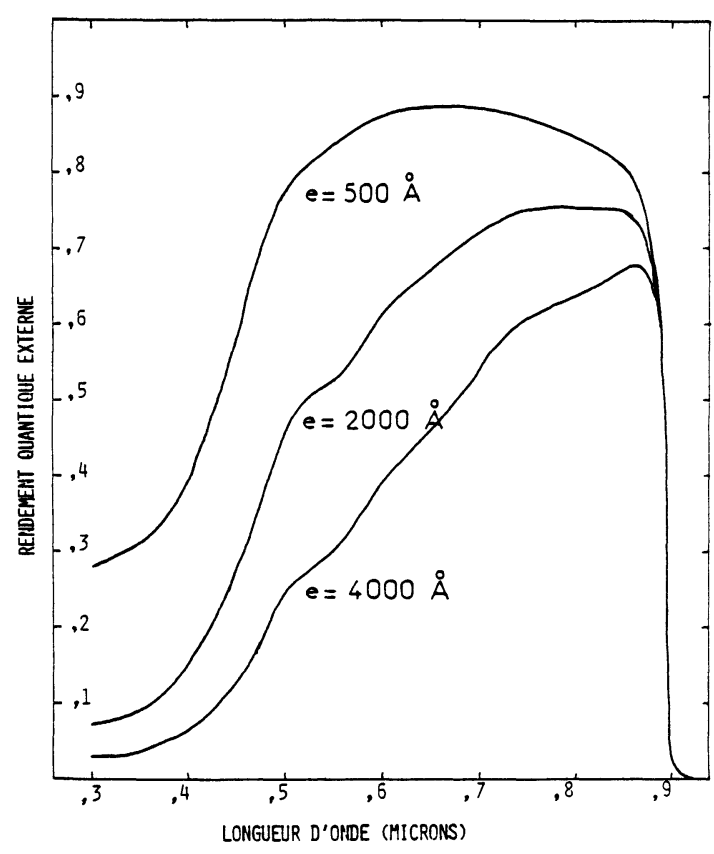

Fig. 2. - Rendement de collecte calculé pour différentes profondeurs de jonction.

[Quantum efficiency calculated for different junction depths.]

liques nous permettant de contrôler avec une bonne homogénéité les faibles épaisseurs, nous avons choisi d'étudier les possibilités des homostructures AsGa à jonction peu profonde.

Les croissances épitaxiales ont lieu sur des substrats d'arséniure de gallium monocristallins orientés suivant l'axe (100). Ils sont dopés à environ $3 \times 10^{18} \mathrm{~cm}^{-3}$.

L'élaboration de la pile consiste en la croissance de trois couches d'AsGa : (voir Fig. 3).

- Une couche tampon de 2 à $3 \mu \mathrm{m}$ d'épaisseur de niveau de dopage et de type comparable à ceux du substrat ; cette couche est nécessaire pour obtenir une bonne qualité cristalline dans la zone active et y éviter la présence des défauts d'interface.

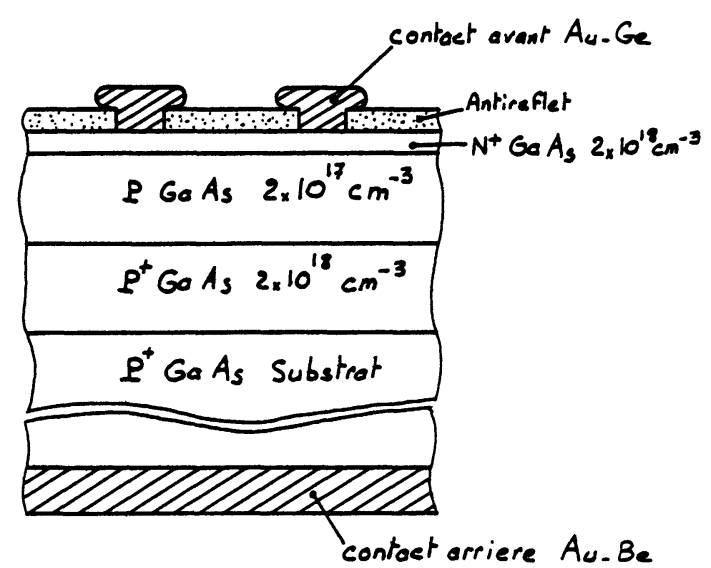

Fig. 3. - Schéma de la photopile.

[Homojunction cell structure.] 
- Une couche d'épaisseur $4 \mu \mathrm{m}$, de même type mais de concentration en porteurs plus faible $\left(10^{16}\right.$ à $10^{18} \mathrm{~cm}^{-3}$ ) que la précédente; cette couche est dite active car la quasi-totalité de l'absorption y aura lieu.

- Une couche très mince, d'épaisseur inférieure à $0,05 \mu \mathrm{m}$, de type opposé et dopé à environ $3 \times 10^{18} \mathrm{~cm}^{-3}$; cette couche est destinée à créer la jonction mais son épaisseur doit être suffisamment faible afin d'y diminuer l'absorption pour éviter les pertes par recombinaison de surface.

Les contacts métalliques sont pris par évaporation d'un alliage d'or ( $\mathrm{Au}-\mathrm{Ge}$ sur le type $\mathrm{N}$ et $\mathrm{Au}-\mathrm{Be}$ sur le type $P$ ) sur les deux faces de la photopile, la grille en face avant étant ensuite gravée par des méthodes classiques de photogravure.

Une couche antireflet, d'épaisseur environ $0,1 \mu \mathrm{m}$, est créée par oxydation anodique de l'arséniure de gallium dans une solution de glycol et d'eau [11]. Nous effectuons les épitaxies sur des substrats de forme carrée et de dimension $1 \mathrm{~cm}^{2}$ sur lesquels, pour étudier l'homogénéité de notre technologie, nous réalisons deux photopiles. De ce fait, la surface de chaque photopile reste faible $\left(0,2 \mathrm{~cm}^{2}\right)$.

3. Résultats expérimentaux. - Deux méthodes de mesure sont utilisées pour caractériser les photopiles :

- le relevé de la sensibilité spectrale, en utilisant un monochromateur et un étalon de puissance,

- le tracé de la caractéristique courant-tension sous l'éclairage d'un simulateur solaire dont la puissance lumineuse est réglée à partir d'une photopile étalon.

La photopile de référence de même technologie que les piles à mesurer et qui porte ci-dessous le numéro 1173 a été étalonnée au soleil, suivant les normes européennes [12], pour un spectre solaire dit «AM1, 5» et dans un laboratoire du Massachusetts Institute of Technology par rapport à une autre pile solaire à l'arséniure de gallium. Ces deux mesures ont conduit au même résultat : $J_{\mathrm{cc}}=24 \mathrm{~mA} / \mathrm{cm}^{2}$.

Les mesures sous simulateur solaire sont ainsi réalisées pour un éclairement équivalent de $100 \mathrm{~mW}$ / $\mathrm{cm}^{2} \mathrm{AM} 1,5$ et une température de $20^{\circ} \mathrm{C}$. Le tracé de la caractéristique $I-V$ (Fig. 4) permet de déterminer les paramètres principaux de chaque dispositif : la densité de photocourant $\left(J_{\mathrm{cc}}\right)$, la tension en circuit ouvert $\left(V_{\text {co }}\right)$, le facteur de forme $(F F)$ et le rendement de conversion $(\eta)$. Le photocourant et le rendement sont calculés par rapport à la surface active de la pile solaire. Ces résultats reportés tableau $I$, font ressortir une certaine dispersion sur les facteurs de forme. Celle-ci est attribuable à une résistance série élevée $\left(1 \Omega \mathrm{cm}^{2}\right.$ à $3 \Omega \mathrm{cm}^{2}$ ) due à une mauvaise optimisation de la géométrie de la grille en face avant.

Un des avantages des photopiles à l'arséniure de gallium pour une utilisation spatiale est leur bon comportement à des températures élevées. Nous avons donc mesuré le rendement de conversion d'une de

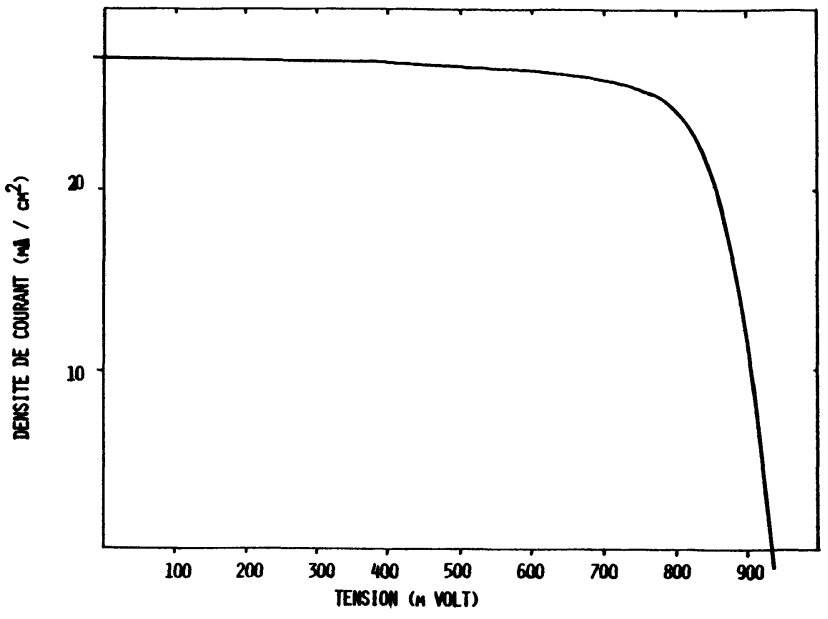

Fig. 4. - Caractéristique courant-tension sous un éclairement correspondant à $100 \mathrm{~mW} / \mathrm{cm}^{2} \mathrm{AM} 1,5$.

[Current-voltage characteristic.]

Tableau I. - Propriétés des photopiles AsGa mesurées sous un éclairement de $100 \mathrm{~mW} / \mathrm{cm}^{2}$ AM1, 5 .

[Device characteristics under AM1, 5 solar illumination.]

$\begin{array}{ccccc}\begin{array}{c}\text { Numéro } \\ \text { photopile }\end{array} & \begin{array}{c}I_{\text {cc }} \\ \left(\mathrm{mA} / \mathrm{cm}^{2}\right)\end{array} & \begin{array}{c}\boldsymbol{V}_{\text {co }} \\ (\mathrm{mV})\end{array} & \begin{array}{c}F F \\ (\%)\end{array} & \begin{array}{c}\eta \\ (\%)\end{array} \\ - & - & - & - & - \\ 1173 & 24,0 & 945 & 74,8 & 17,0 \\ 1333 & 26,6 & 891 & 78,2 & 18,5 \\ 1603 & 27,2 & 931 & 77,0 & 19,5 \\ 1604 & 28,0 & 924 & 73,2 & 18,9 \\ 1607 & 26,0 & 932 & 76,1 & 18,5 \\ 1609 & 27,3 & 936 & 76,7 & 19,6\end{array}$

ces piles (1603) de la température ambiante jusqu'à $120^{\circ} \mathrm{C}$. Les résultats sont portés sur la figure 5 en valeurs relatives afin de pouvoir les comparer à ceux obtenus à partir d'une photopile classique au silicium [13].

La figure 6 montre les variations des paramètres $J_{\mathrm{cc}}, V_{\mathrm{co}}, F F$ et $\eta$ en fonction de la température.

Nous voyons ainsi que la densité de courant augmente avec la température à raison de $0,025 \mathrm{~mA} /$ $\mathrm{cm}^{2} /{ }^{\circ} \mathrm{C}$; cette croissance est due en partie au déplacement vers les grandes longueurs d'onde du front d'absorption. Les autres paramètres par contre diminuent la tension en circuit ouvert de $-2,17 \mathrm{mV} /{ }^{\circ} \mathrm{C}$, le facteur de forme de $-0,06 \% /{ }^{\circ} \mathrm{C}$, et le rendement avec un taux moyen de $-0,045 \% /{ }^{\circ} \mathrm{C}$. Ces valeurs sont strictement comparables à celles obtenues par la NASA sur des photopiles à hétéroface GaAlAs [14].

La caractérisation des photopiles ne serait pas complète sans un relevé de la sensibilité spectrale (Fig. 7). En effet, l'analyse du rendement de collecte permet de mettre en évidence les phénomènes qui limitent le photocourant. Cette analyse se trouve facilitée par une simulation sur ordinateur réalisée à partir du modèle de Hovel [15]. En particulier, 


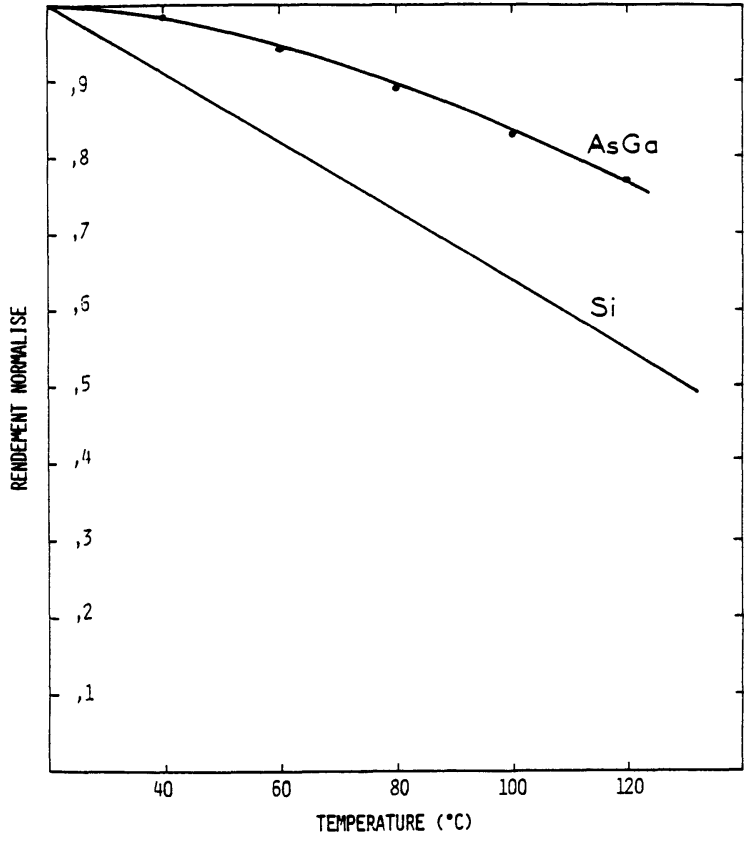

Fig. 5. - Comparaison de l'évolution du rendement de conversion en fonction de la température d'une photopile silicium et d'une photopile AsGa.

[Effect of temperature on conversion efficiency for both $\mathrm{Si}$ and $\mathrm{GaAs}$ cells.]

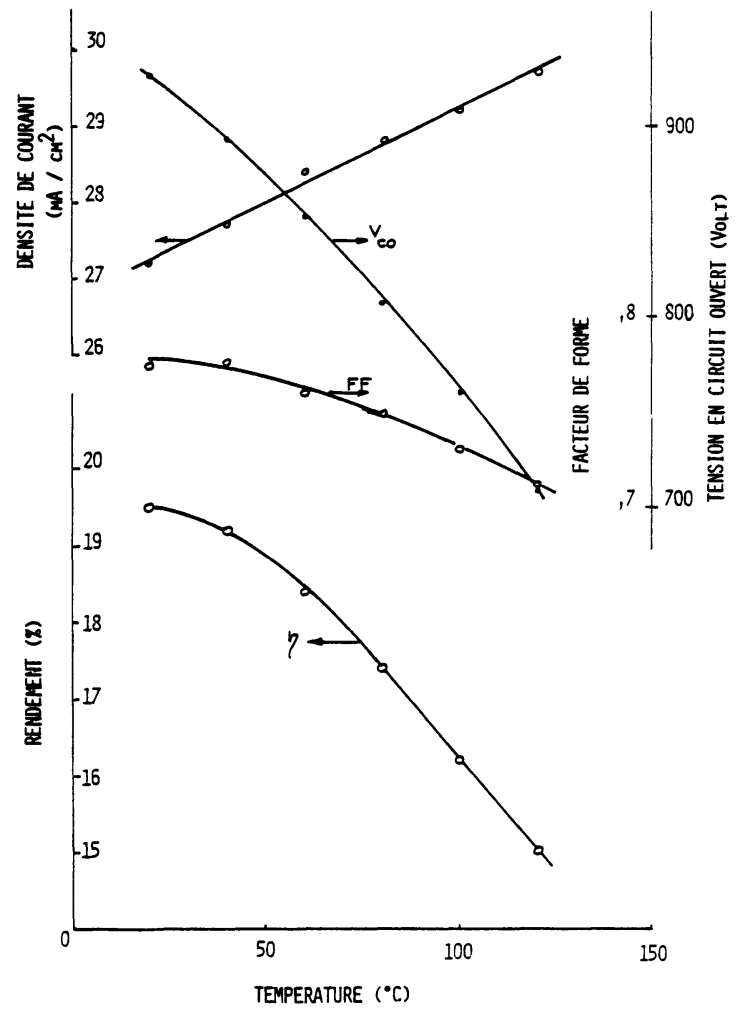

Fig. 6. - Influence de la température sur les paramètres principaux de la photopile.

[Physical parameters of GaAs cell as a function of temperature.]

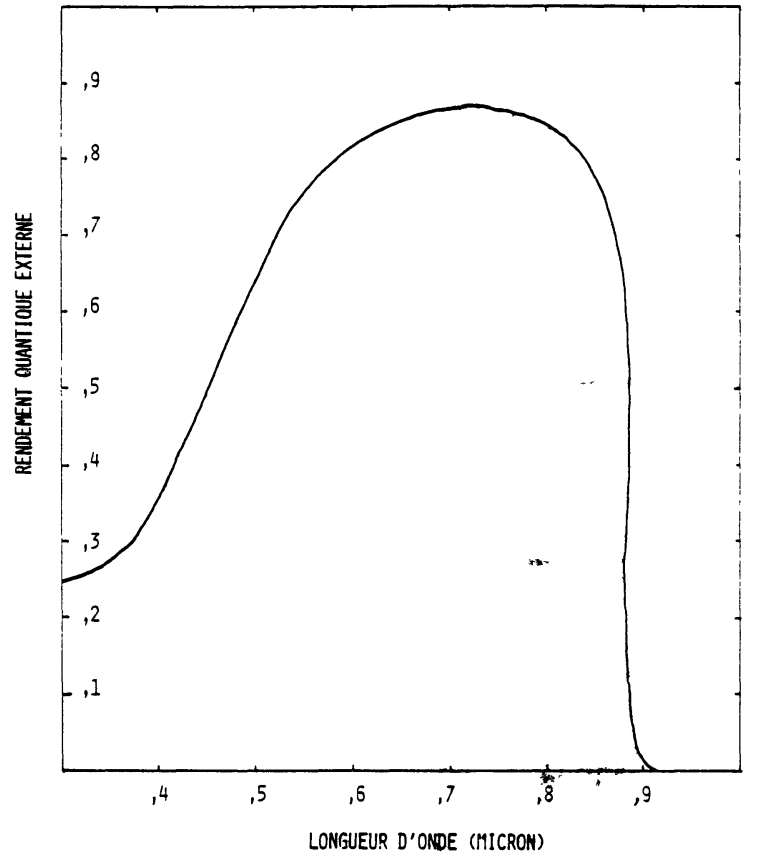

Fig. 7. - Rendement de collecte externe.

[External quantum efficiency]

il nous est possible par cette simulation d'estimer certaines caractéristiques des photopiles, telles que la vitesse de recombinaison en surface et la longueur de diffusion dans la couche active.

Si nous avons peu de possibilité pour améliorer le premier de ces paramètres, (notre seule parade consistant à diminuer la profondeur de jonction), nous pouvons par contre intervenir sur la longueur de diffusion en modifiant le taux de dopage dans la couche active et même le type du dopant. Les valeurs ainsi déterminées sont indiquées dans le tableau II.

Tableau II. - Longueur de diffusion dans la zone active des photopiles déterminée à partir de la réponse spectrale.

[Diffusion length characterized by spectral response.]

$\begin{array}{ccccc}\begin{array}{c}\text { Numéro } \\ \text { photopile }\end{array} & \text { Type } & \begin{array}{c}\text { Couche active } \\ \text { Dopant }\end{array} & \begin{array}{c}\text { Dongueur } \\ \text { de diffusion } \\ (\mathrm{cm})\end{array} \\ 1173 & \frac{\mathrm{P}}{-} & - & - & - \\ 1333 & \mathrm{~N} & \mathrm{Zn} & 4 \times 10^{17} & 6 \times 10^{-4} \\ 1603 & \mathrm{~N} & \mathrm{Ge} & 6 \times 10^{16} & 3 \times 10^{-4} \\ 1604 & \mathrm{~N} & \mathrm{Ge} & 3 \times 10^{16} & 5 \times 10^{-4} \\ 1607 & \mathrm{~N} & \mathrm{Ge} & 5 \times 10^{16} & 3 \times 10^{-4} \\ 1609 & \mathrm{~N} & \mathrm{Se} & 8 \times 10^{-4} & 5 \times 10^{-4}\end{array}$

Enfin, nous avons entrepris un programme d'étude du comportement de nos photopiles en ambiance spatiale. Cette étude durera plusieurs mois, mais déjà les premières mesures laissent espérer que les résultats seront très favorables à ce type de structure.

La photopile 1607 a été soumise à un flux de $3 \times 10^{15}$ électrons de $1 \mathrm{MeV}$ par $\mathrm{cm}^{2}$. Ce flux simule 


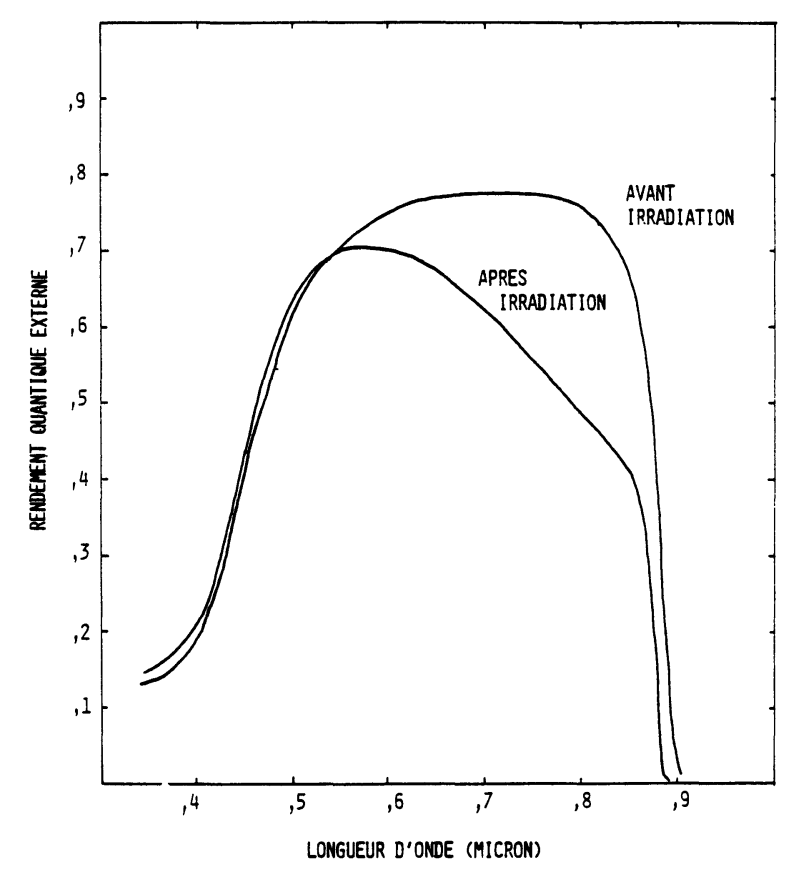

Fig. 8. - Evolution du rendement de collecte sous l'effet de l'irradiation par électrons de $1 \mathrm{MeV}$.

[Quantum efficiency as a function of $1 \mathrm{MeV}$ electron fluence.]

les conditions spatiales d'un satellite géostationnaire de 7 ans de durée de vie. Les figures 8 et 9 illustrent les résultats obtenus :

- Sur la figure 8 sont portés les rendements de collecte avant et après irradiation; comme pour les photopiles au silicium, il s'agit de la réponse dans la partie rouge du spectre qui est affectée par l'irradiation ; la simulation sur ordinateur nous apprend que ceci est justifié par une dégradation de la longueur de diffusion dans la couche active qui est passée de $3 \mu \mathrm{m}$ avant irradiation à $0,4 \mu \mathrm{m}$ après.

- La figure 9 représente l'évolution en cours d'irradiation du rendement photovoltaïque normalisé

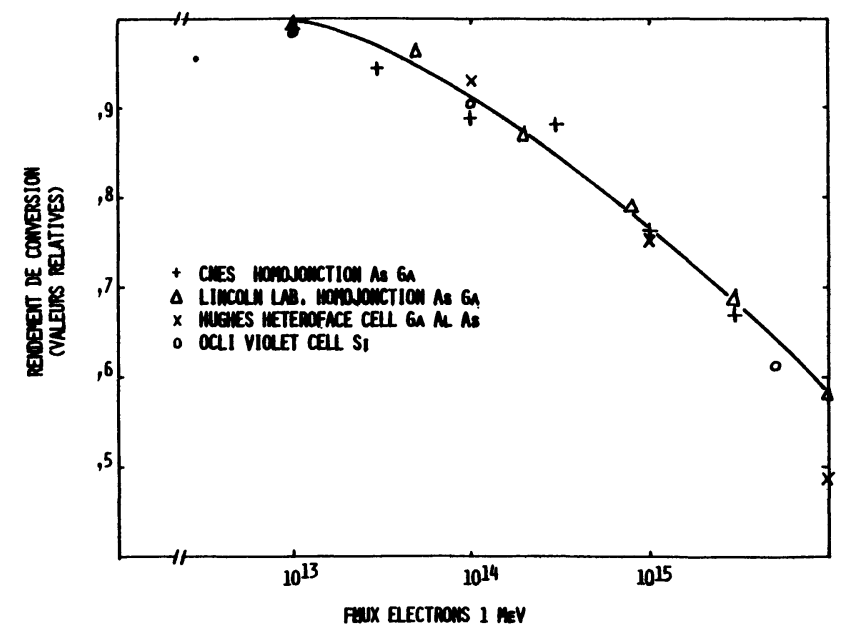

Fig. 9. - Evolution du rendement photovoltaïque sous l'effet de l'irradiation par électrons de $1 \mathrm{MeV}$.

[Conversion efficiency versus $1 \mathrm{MeV}$ electron fluence.]

de notre photopile; à titre comparatif est indiquée la dépendance de trois autres types de photopiles telle qu'elle est reportée par J. C. C. Fan et al. [4].

4. Conclusion. - Les rendements de conversion photovoltaiqque élevés que nous avons obtenus sur les homojonctions AsGa préparées par CVD-OM sont comparables aux performances atteintes par les photopiles GaAlAs/GaAs réalisées dans d'autres laboratoires par d'autres techniques épitaxiales.

L'aptitude de ces structures à fonctionner à plus haute température que les piles solaires au silicium et leur résistance aux radiations les rendent attrayantes pour les utilisations spatiales.

L'épitaxie en phase vapeur aux organométalliques démontre ainsi son efficacité à réaliser des matériaux d'excellente qualité et apparaît déjà comme une technique bien adaptée à la fourniture de photopiles pour les missions spatiales.

\section{Bibliographie}

[1] Woodall, J. M., Hovel, H. J., Appl. Phys. Lett. 30 (1977) 492.

[2] Loo, R., Goldhammer, L., ANSPauch, B., KNEChtli, T. C., Kamath, G. S., 13 th IEEE Photov. Special. Conf. 1978, p. 562.

[3] GaAlAl/GaAs Solar Cell Process Study. NASA Contractor Report 3361 (1980).

[4] Fan, J. C. C., Chapman, R. L., Bozler, C. O., Appl. Phys. Lett. 36 (1980) 54.

[5] Manasevit, G. M. and Simpson, W. I., J. Electrochem. Soc. 116 (1969) 1725.

[6] DuPUIS, R. D. et al., 14th IEEE Photovoltaic Special. Conf. 1980, p. 1388.

[7] Saxena, T. T. et al., J. Appl. Phys. 51 (1980) 4501.

[8] Gave, G., Bouree, J. E., Lemetayer, M., Dorio, P., Revue Phys. Appl. 14 (1979) 875.

[9] Gave, G., Bouree, J. E., Lemetayer, M., Dorio, P., 14th IEEE Photovoltaic Special. Conf. 1980, p. 360.
[10] Bozler, C. O., Fan, J. C. C., Appl. Phys. Lett. 31 (1977) 629.

[11] Hasegawa, H., Hartnagez, H. L., J. Electrochem. Soc. 123 (1976) 713.

[12] Standard Procedures for Terrestrial Photovoltaic Performance Measurements. Commission of the European Communities. Specification $\mathrm{n}^{\mathrm{o}} 101, \mathrm{Feb}$. 1979.

[13] Kamath, G. S., 16th Intersoc. Energ. Conv. Engin. Conf. 1981, p. 416.

[14] Walker, G. H., Conway, J. E., Hong, K. G., HeinBOCKEL, J. H., 14th IEEE Photovoltaic Special. Conf. 1980, p. 1098.

[15] Hovel, H. J., Semiconductors and Semimetals, Vol. 11, Solar Cells (Academic Press, New York) 1975, p. 15. 